\title{
Dysregulation of Dopamine and Glutamate Release in the Prefrontal Cortex and Nucleus Accumbens Following Methamphetamine Self-Administration and During Reinstatement in Rats
}

\author{
Aram Parsegian' and Ronald E See ${ }^{*, 1,2}$ \\ 'Department of Neurosciences, Medical University of South Carolina Charleston, Charleston, SC, USA; ${ }^{2}$ Faculty of Medicine, \\ University of Tabuk, Tabuk, Saudi Arabia
}

\begin{abstract}
Methamphetamine (meth) addicts often exhibit enduring cognitive and neural deficits that likely contribute to persistent drug seeking and the high rates of relapse. These deficits may be related to changes in the prefrontal cortex (PFC) and its glutamatergic projections to the nucleus accumbens (NAc). Here, we performed in vivo microdialysis in the PFC and NAc in rats following either meth self-administration or yoked-saline control histories to assess baseline glutamate $(G L U)$ levels, or reinstatement-evoked GLU and dopamine (DA) efflux in both regions simultaneously under cue-induced, meth-primed, or combined cues + meth reinstatement conditions. Our results show that meth self-administration (I) reduced basal GLU levels in both the dmPFC and NAc, (2) concurrently increased dmPFC and NAc GLU efflux during reinstatement, and (3) increased DA efflux in the dmPFC, but not in the NAc, under all reinstatement conditions when compared with yoked-saline controls. These data demonstrate for the first time that a history of psychostimulant self-administration alters GLU homeostasis not only in the NAc, but also in the dmPFC, its primary GLU projection source. Furthermore, combined cues + meth-primed reinstatement conditions produced the most pronounced increases in mPFC and NAc extracellular GLU, suggesting that the cue and meth prime conditions are additive in promoting reinstatement. Finally, increased efflux of DA in the dmPFC, but not in the NAc, across reinstatement conditions suggests that DA release in the dmPFC may be an important mediator of drug seeking initiated by multiple relapse triggers.

Neuropsychopharmacology (2014) 39, 8II-822; doi: 10.1038/npp.2013.231; published online 9 October 2013
\end{abstract}

Keywords: dopamine; glutamate; methamphetamine self-administration; nucleus accumbens; prefrontal cortex; reinstatement

\section{INTRODUCTION}

The development of drug addiction is believed to derive in large part from maladaptive neurobiological responses to drugs of abuse within the corticostriatal glutamate (GLU) and mesostriatal dopamine (DA) systems of the brain (Berridge and Robinson, 1998; Wise, 2004; Kalivas et al, 2005). Although these circuits normally optimize behavior in response to a changing environment and establish welllearned behaviors, their dysregulation due to repeated drug abuse likely underlies the switch to habitual drug-taking behaviors, which operate outside of normal cortical regulation (Goldstein et al, 2009; George and Koob, 2010).

Methamphetamine (meth) addicts often exhibit pronounced deficits in cortical regulation of subcortical drive (Baicy and London, 2007; Salo et al, 2009). The impact of

*Correspondence: Dr RE See, Faculty of Medicine, University of Tabuk, PO Box 74I, Tabuk 7|49I, Saudi Arabia, Tel: +966056 619 9409, Fax: +966044262 579, E-mail: rsee@ut.edu.sa

Received 17 June 2013; revised 8 August 2013; accepted 27 August 2013; accepted article preview online 2 September 2013 meth on this circuitry may be more profound than other addictive drugs, due in part to its long half-life, high-lipid solubility, fast uptake and accumulation, and distinctive pharmacological effects on both DA and GLU functions (Bowyer et al, 2008; Fowler et al, 2008; Stephans and Yamamoto, 1994). Thus, meth abuse may alter DA receptive pyramidal neurons in the PFC, in turn affecting downstream signaling in the nucleus accumbens core (NAc). DA projections to the dmPFC and NAc arise primarily from the ventral tegmental area (VTA) and facilitate the establishment of conditioned reinforcement, reward learning, and addictive behavior (Berridge and Robinson, 1998; Schultz, 2007). As DA signaling in this circuitry is central for reward processing, meth-induced supraphysiological increases in DA likely promote the maladaptive changes that underlie the transition to compulsive drug use (Di Chiara, 1999; Wise, 2004). Specifically, an aberrant degree of DA-reinforced learning may contribute to 'over learning' of drug-associated memories and ingraining incentive salience to the rewarding physiological effects and cues associated with drug taking, both of which substantially contribute to the propensity for relapse (Berridge and Robinson, 1998; Wise, 2004). 
Similarly, GLU transmission in the corticostriatal pathway is necessary for the expression of motivated behaviors associated with abused drugs. The GLU projection from the dmPFC to the NAc is critical for reinstatement of drug seeking across several drugs of abuse (McFarland et al, 2003; Kalivas et al, 2005), including meth (Rocha and Kalivas, 2010). Moreover, the relationship between extrasynaptic and synaptic basal GLU in the NAc has an important role in reinstatement of extinguished cocaine seeking (Cornish and Kalivas, 2000; Kalivas, 2009). Both reduced basal GLU levels and cocaine-induced GLU increases in the NAc critically underlie the reinstatement of cocaine seeking (Baker et al, 2003; McFarland et al, 2003; Kalivas, 2009). However, whether meth self-administration similarly affects GLU in the NAc or induces such changes in GLU homeostasis upstream in the dmPFC has not been determined. Indeed, no published studies with any drugs of abuse have previously examined GLU release in the dmPFC in the basal state or during reinstatement of drug seeking after a history of drug self-administration.

Several neuromodulatory monoamines, particularly DA, converge onto cortical GLU projection neurons and are clearly impacted during repeated meth exposure (Haughey et al, 2000; Shoblock et al, 2003). As DA is important for acquiring both primary and secondary reward associations (Schultz, 2007), repeated experience with meth intake influences both of these processes. Prior studies have shown that different neuroanatomical inputs and therefore neurotransmitter signals can contribute to cue-induced $v s$ drug-primed drug seeking (Fuchs et al, 2004; McLaughlin and See, 2003). Importantly, although drug-associated cues or a meth-prime independently elicit drug seeking, whether DA or GLU release in the dmPFC or NAc shows the same pattern under these two conditions, or whether they are additive when combined has not been tested.

Here, we report on the first concurrent assessment of the dmPFC and NAc following a history of meth self-administration and extinction of responding in order to determine whether: (a) basal GLU levels were altered in the dmPFC and NAc, (b) GLU efflux in these regions was differentially activated during cue-induced, meth-primed, or cues + meth-primed reinstatement of meth seeking, and (c) DA efflux in these two regions varied under these different reinstatement conditions.

\section{MATERIALS AND METHODS}

\section{Subjects}

One hundred and ten adult male Long-Evans rats weighing $300-325 \mathrm{~g}$ at the time of delivery from Charles River Laboratories (Wilmington, MA) were maintained on a reversed $12: 12 \mathrm{~h}$ light/dark cycle in a temperature- and humidity-controlled vivarium. Rats were individually housed and received water ad libitum throughout the study and $25 \mathrm{~g}$ standard rat chow (Harlan, Indianapolis, IN) daily until self-administration stabilized, at which time rats were maintained ad libitum for both food and water. Procedures were conducted in accordance with the Guide for the Care and Use of Laboratory Rats (Institute of Laboratory Animal Resources on Life Sciences, National Research Council, Washington, DC; 1996) and approved by the Institutional
Animal Care and Use Committee of the Medical University of South Carolina.

\section{Surgery}

Rats were anesthetized i.p. with ketamine $(66 \mathrm{mg} / \mathrm{kg})$, xylazine $(1.3 \mathrm{mg} / \mathrm{kg})$, and Equithesin $(0.5 \mathrm{ml} / \mathrm{kg})$. An intravenous silastic catheter (Dow Corning; inner diameter, $0.5 \mathrm{~mm}$; outer diameter, $0.9 \mathrm{~mm}$ ) constructed using previously described methods (Reichel et al, 2011) was surgically implanted into the right jugular vein and secured with silk sutures. The catheter was then passed subcutaneously to the top of the rat's back posterior to the shoulder blades. This end was attached to an infusion harness (Instech Laboratories) that provided access to an external port for intravenous drug delivery. An antibiotic solution of cefazolin $(10 \mathrm{mg} / 0.1 \mathrm{ml})$ was given after surgery and during recovery, along with $0.1 \mathrm{ml}$ of $70 \mathrm{U} / \mathrm{ml}$ heparinized saline.

Immediately after catheterization, while the rats were maintained under anesthesia, two stainless steel guide cannulae (20 gauge) were implanted using the following stereotactic coordinates: dmPFC (targeting the prelimbic cortex) with AP $+3.2 \mathrm{~mm}, \mathrm{ML} \pm 0.6 \mathrm{~mm}$ from bregma, and DV $-2.5 \mathrm{~mm}$ from dura; NAc (targeting the core) with AP $+1.3 \mathrm{~mm}, \mathrm{ML} \pm 3.0 \mathrm{~mm}$ at a $10^{\circ}$ angle from bregma, and DV $-6.1 \mathrm{~mm}$ relative to dura (Paxinos and Watson, 1997). All cannula placements were counterbalanced (in the right or left hemisphere) to avoid any lateralization bias, and wire stylets were placed in the guide cannulae to prevent clogging.

\section{Meth Self-Administration and Extinction}

After at least 5 days of recovery, rats were given daily $2 \mathrm{~h}$ sessions to self-administer meth along a fixed ratio 1 schedule of reinforcement. A house light signaled the beginning of each session and remained on throughout the session. During the session, a response on the active lever resulted in the activation of a pump for a $2 \mathrm{~s}$ infusion of meth $(20 \mu \mathrm{g} / 50 \mu \mathrm{l}$ bolus, i.v. $)$, followed by a $20 \mathrm{~s}$ time-out period to prevent overdose. Each infusion was paired with a tone $(78 \mathrm{~dB}, 4.5 \mathrm{kHz})$ and a white stimulus light over the active lever that came on as soon as the infusion occurred and lasted for $5 \mathrm{~s}$. Responding during time-out or on the inactive lever was recorded, but resulted in no programmed consequences. Yoked-saline controls were attached to an infusion line identical to that used for meth and received $50 \mu \mathrm{l}$ infusions of $0.9 \%$ saline and the tone + light stimulus during daily $2 \mathrm{~h}$ sessions whenever the matched selfadministering subject received a meth infusion. After each session, catheters were flushed with cefazolin and $0.1 \mathrm{ml}$ of $70 \mathrm{U} / \mathrm{ml}$ heparinized saline. Catheter patency was periodically verified with methohexital sodium $(10 \mathrm{mg} / \mathrm{ml}$ dissolved in $0.9 \%$ physiological saline), a short acting barbiturate that produces a rapid loss of muscle tone. Self-administration was followed by 10 daily extinction sessions, where active lever pressing no longer resulted in any programmed consequences.

\section{Microdialysis}

No net flux microdialysis. Rats were placed in a novel sound-attenuated, drug-neutral chamber overnight to 
acclimate, whereas the probes were perfused with a perfusion medium at $0.2 \mu \mathrm{l} / \mathrm{min}$ overnight $(\sim 16 \mathrm{~h})$. Perfusion medium consisted of modified Ringer's solution (in mM: $\mathrm{NaCl}, 147 ; \mathrm{KCl}, 2.7 ; \mathrm{MgCl}_{2}, 1.0 ; \mathrm{CaCl}_{2}, 1.2 ; \mathrm{Na}_{2} \mathrm{HPO}_{4}$, 0.5 ; final $\mathrm{pH}$ of 7.4). The following morning, the perfusion rate was increased to $2.0 \mu \mathrm{l} / \mathrm{min}$ and a $2 \mathrm{~h}$ baseline was collected. Perfusate was then progressively switched to a modified Ringer's solution containing $0.5,1.25,2.5$, and $5.0 \mu \mathrm{M}$ of GLU. These concentrations were reverse dialyzed into either the dmPFC or NAc and the output dialysate was collected separately every $20 \mathrm{~min}$. The dmPFC and NAc were assessed on separate days in a counterbalanced order and the rats were allowed 2 days to recover between tests. Collected samples were immediately frozen and kept at $-80{ }^{\circ} \mathrm{C}$ until high performance liquid chromatography (HPLC) analysis. Following this experiment, animals were deeply anesthetized with an overdose of Equithesin and underwent transcardial perfusion with $4 \%$ paraformaldehyde. Each brain was preserved for histology. Rats who failed to reach the self-administration criterion or who showed inaccurate histology were excluded from final analysis.

Reinstatement microdialysis. Following a $2 \mathrm{~h}$ baseline collection, subjects received either a meth prime $(1 \mathrm{mg} / \mathrm{kg}$, i.p.) or vehicle prime (saline, i.p.) injection and were placed in the self-administration chamber with or without contingent tone + light cues and allowed to lever press for $2 \mathrm{~h}$. Microdialysis samples were obtained every $20 \mathrm{~min}$, immediately frozen, and stored at $-80{ }^{\circ} \mathrm{C}$. Following reinstatement microdialysis, subjects were deeply anesthetized, transcardially perfused, and their brains were preserved for histology. Subjects that failed to reach selfadministration criteria or showed inaccurate histology were excluded from final analysis.

High performance liquid chromatography. At the time of analysis, stored samples were thawed and aliquots placed into sample vials for HPLC. For determination of GLU, precolumn derivatization of GLU with O-phthaldelhyde was performed using a Shimadzu Autosampler Model SIL10AXL. Mobile phase consisted of 35\% methanol (v/v), 5\% acetonitrile $(\mathrm{v} / \mathrm{v}), 100 \mathrm{mM} \mathrm{Na}_{2} \mathrm{HPO}_{4}, \mathrm{pH}=6.75$. GLU was separated using a reverse-phase column $(3.0 \mu \mathrm{m}$, $100 \times 4.6 \mathrm{~mm}$, Luna $3 \mathrm{u} \mathrm{C18(2),} \mathrm{Phenomenex)} \mathrm{and} \mathrm{detected}$ by a fluorescence detector (Shimadzu) with an excitation wavelength of $337 \mathrm{~nm}$ and emission wavelength of $545 \mathrm{~nm}$. For determination of DA, samples were injected by an autosampler (Dionex-ESA model 543, Chelmsford, USA) onto a reverse-phase column (MD150, ESA) maintained at $30^{\circ} \mathrm{C}$ and detected by an electrochemical detector (ESA Coulochem III). Mobile phase consisted of $90 \mathrm{~mm}$ sodium dihydrogen phosphate monobasic, $50 \mathrm{~mm}$ citric acid, $1.7 \mathrm{mM}$ 1-octanesulfonic acid, $50 \mu \mathrm{M}$ EDTA, and $9 \%$ acetonitrile (final $\mathrm{pH} 3$ ). Dialysate concentrations for DA and GLU were determined by comparing peak areas with that of a standard curve. Data were analyzed by computerized chromatography analysis systems (Agilent EZChrome Elite and Shimadzu). For the no net flux experiment, subtracting output from input GLU concentrations created a plotted curve of the calculated concentrations. The point

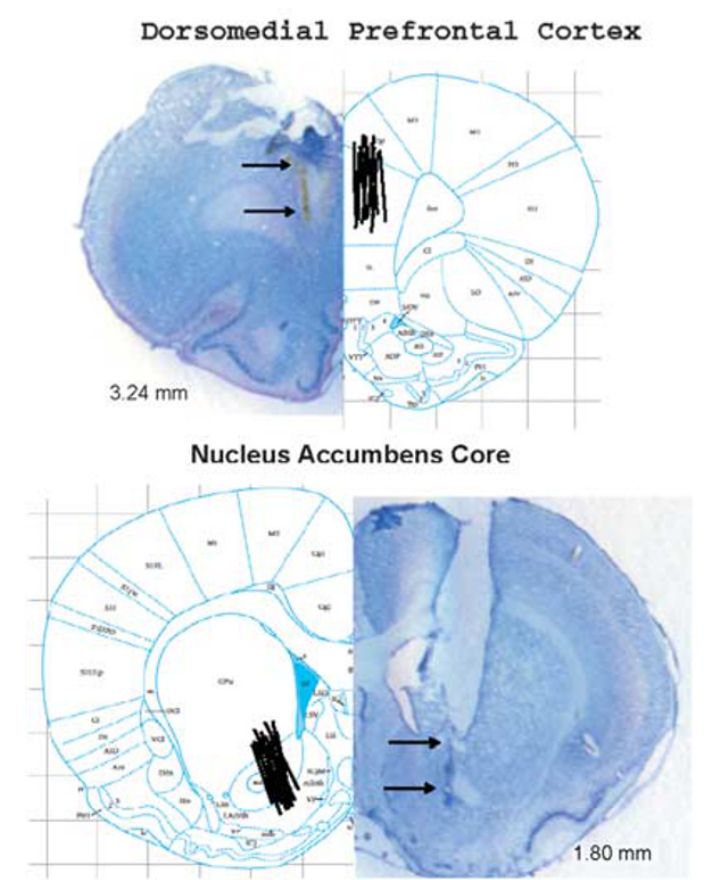

Figure I Location of microdialysis probes for 15 randomly selected dialysis tracks through the dmPFC and NAc. Arrows indicate the extent of the microdialysis probes ( $2 \mathrm{~mm}$ length) into the dmPFC (top) and into the NAc core at a $10^{\circ}$ angle (bottom). The atlas images are adapted from (Paxinos and Watson, 1997), and AP coordinates (in mm) are given relative to bregma.

of no net change in concentration ( $x$-intercept) was used to define basal GLU levels in each brain region (Lonnroth et al, 1987). For the reinstatement microdialysis experiments, calculated GLU and DA concentrations were averaged across subjects by condition (meth $v s$ yoked saline) in $20 \mathrm{~min}$ bins and expressed as a percent of the averaged baseline over the course of reinstatement testing.

\section{Histology}

Immediately following the completion of each microdialysis experiment, rats were deeply anesthetized, transcardially perfused with $4 \%$ paraformaldehyde, and decapitated. The brains were cut and stained using cresyl violet for histological verification of the cannulae and probe tracts (Figure 1).

\section{Data Analysis}

Data were analyzed using one-way or two-way analysis of variance (ANOVA), linear regression, or $t$-tests, where appropriate. The alpha level for all analyses was set at $p=0.05$. For no net flux microdialysis, linear regression analysis was used to compare calculated slopes and points of no net flux ( $x$-intercepts) between subjects with meth or yoked-saline histories. All data were analyzed using GraphPad Prism 6 (GraphPad Software, La Jolla, CA). Lever responding during the last 3 days of extinction and during reinstatement was analyzed with mixed-factor two-way ANOVA with a between-subjects factor of reinstatement condition (control, cue-induced, meth-primed, and cues + meth-primed) and repeated measures (RM) over time 
(extinction $v s$ reinstatement). Pairwise comparisons between individual reinstatement groups were conducted using the Tukey's test, where appropriate. Reinstatement microdialysis data were transformed to percent of baseline and a mean baseline value was compared with subsequent samples during reinstatement using separate two-way RM ANOVA with a between-subjects factor of drug history (meth $v s$ yoked saline) and repeated measurements over time (20 min bins). Pairwise comparisons between individual time points were conducted using Bonferroni's multiple comparison tests, where appropriate.

\section{RESULTS}

\section{Meth Self-Administration and Extinction}

Rats self-administered meth for $2 \mathrm{~h}$ /day over 10 days or received yoked non-contingent saline vehicle (yoked control). Average active lever responses for the last 3 days of self-administration for each condition (mean \pm SEM) were as follows: no net flux $(45.08 \pm 9.37)$, no cues or prime (42.50 \pm 7.06$)$, cue-induced (43.22 \pm 8.44$)$, meth-primed $(38.90 \pm 5.30)$, and cues + meth-primed $(43.67 \pm 6.86)$. Total meth intake $(\mathrm{mg} / \mathrm{kg})$ over 10 days of self-administration for each condition was as follows: no net flux $(21.78 \pm 1.67)$, control (21.68 \pm 1.38$)$, cue-induced (22.24 \pm 1.41$)$, methprimed $(21.47 \pm 1.03)$, and cues + meth-primed (23.70 \pm 1.83). All rats reached the extinction criterion of $<20$ active lever presses for the last 3 days of extinction before subsequent testing. The following day, rats underwent either no net flux microdialysis testing or standard microdialysis during reinstatement testing.

\section{Meth Self-Administration Reduced Basal GLU Levels in the dmPFC and NAc}

Figure 2 shows that basal GLU levels were significantly reduced in the $\mathrm{dmPFC}$ and NAc in rats with a prior history of meth self-administration. Linear regression analysis was used to calculate both the slope and values of the $x$-intercept, a measure that indicates basal extracellular GLU levels within the dmPFC and NAc. Although the slopes in the $\mathrm{dmPFC}$ and NAc were not different $(\mathrm{F}(1,80)=3.52$, $p=0.06$ and $\mathrm{F}(1,76)=0.30, p=0.59$, respectively), $x$-intercept data showed that extracellular GLU levels were significantly lower both in the $\operatorname{dmPFC}(\mathrm{F}(1,81)=17.06$, $p<0.05)$ and $\operatorname{NAc}(\mathrm{F}(1,77)=30.83, p<0.05)$ in rats with a meth history.

\section{Reinstatement of Meth Seeking}

Separate groups of rats were tested under different conditions for reinstatement of meth seeking (no cues or prime, cue-induced, meth-primed, and cues + methprimed) during microdialysis sampling. Figure 3a shows daily meth self-administration and extinction sessions for the four groups. Rats showed stable meth self-administration and typical extinction curves over time, with no significant differences seen between groups. Figure $3 \mathrm{~b}$ shows cumulative active lever presses (mean \pm SEM) during each reinstatement session compared with the average of the last 3 days of responding during extinction for each
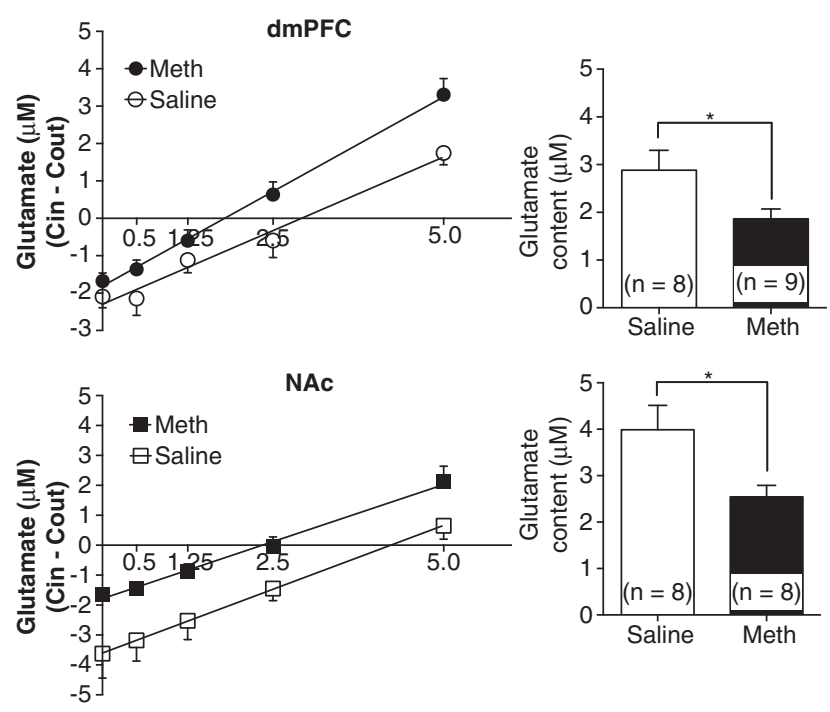

Figure 2 Basal levels of extracellular GLU were significantly lower in both the dmPFC (top) and the NAc (bottom) following meth selfadministration and extinction as compared with yoked-saline controls. Plots from no net flux in vivo microdialysis experiments show the mean gain or loss of dialysate GLU concentrations to and from the brain as a function of the perfusate GLU concentration. Linear regression analysis was used to calculate both the slope and values of the $x$-intercept, a measure that indicated the extracellular GLU levels within the dmPFC and NAc. Although the slopes in the dmPFC and NAc were not significantly different, extracellular GLU levels were significantly lower in the dmPFC and NAc in rats with a history of meth self-administration $(* p<0.05)$.

group. A two-way ANOVA revealed main effects of group $(\mathrm{F}(3,56)=6.95, p<0.05)$ and time (extinction $v s$ reinstatement $)(\mathrm{F}(1,56)=30.03, p<0.05)$, and a significant interaction $(\mathrm{F}(3,56)=6.19, \quad p<0.05)$. As expected, pairwise comparisons showed that cues, meth-primed, and cues + meth-primed conditions produced significant reinstatement of meth seeking (Tukey, $p<0.05$ ). Notably, active lever responding during the cues + meth-primed condition was significantly greater than all other conditions (Tukey, $p<0.05$ ). Figure $3 \mathrm{~b}$ (inset) shows the time line of active lever pressing on the previously meth-paired lever in $20 \mathrm{~min}$ bins across the 2-h reinstatement test (note that binned data do not reflect all subjects, as a few initial collections were not recorded in separate bins; however, this omission was random across conditions). Meth seeking behavior mirrored the GLU efflux in both brain regions for reinstatement triggered by cues, meth-primed, or cues + meth-primed conditions.

Pre-reinstatement basal (uncorrected) neurotransmitter levels (mean \pm SEM) were equivalent in all four reinstatement conditions, with no significant differences found between groups. For DA $(\mathrm{nm})$ in the dmPFC: meth $=$ $0.31 \pm 0.13$, saline $=0.26 \pm 0.02$; in the NAc: meth $=0.86 \pm$ 0.06 , saline $=0.81 \pm 0.05$. For GLU ( $\mu \mathrm{M})$ in the dmPFC: meth $=1.48 \pm 0.11$, saline $=1.68 \pm 0.22$; in the NAc: meth $=$ $1.98 \pm 0.03$, saline $=2.02 \pm 0.34$.

\section{GLU Efflux during Reinstatement}

Figure 4 depicts extracellular GLU over time in the dmPFC during (A) no cues or prime, (B) cue-induced, (C) methprimed, or (D) cues + meth-primed reinstatement of meth 
a

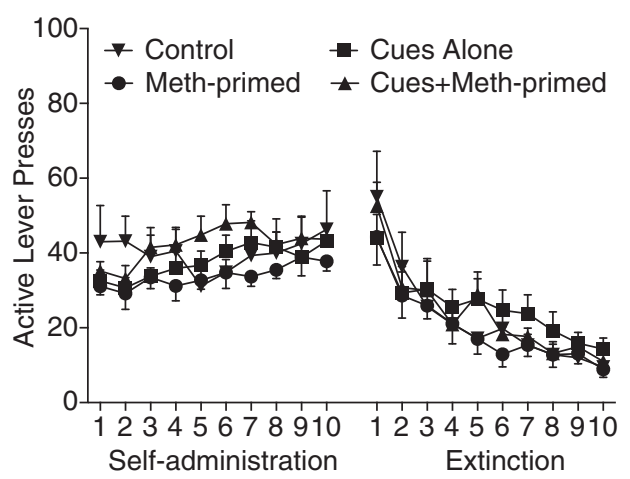

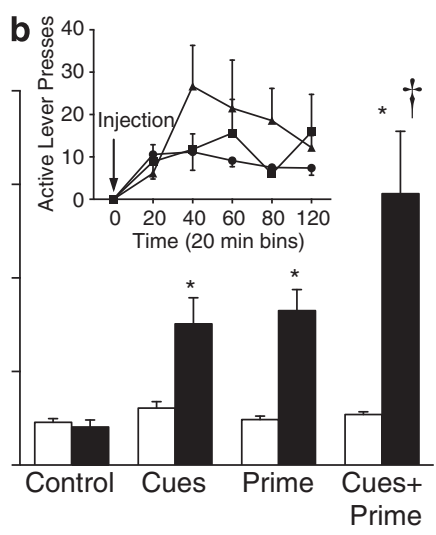

Figure 3 Meth self-administration, extinction, and reinstatement. (a) Daily meth self-administration and subsequent extinction sessions ( $2 \mathrm{~h} /$ day) in the four different reinstatement groups. No differences were seen between groups before reinstatement at any time point. (b) Active lever pressing ( $2 \mathrm{~h}$ session) on the previously meth-paired lever during control (vehicle prime, no cues), cue-induced, meth-primed, and cues + meth-primed reinstatement sessions compared with the extinction responding for each respective condition (open bars). Rats that experienced meth self-administration showed significant reinstatement of meth seeking during cue-induced, meth-primed, and cues + meth-primed reinstatement $(* p<0.05)$. Notably, responding during the cues + meth-primed condition was significantly greater than all other conditions $\left({ }^{\dagger} p<0.05\right)$. Inset: active lever pressing in 20 min bins over the $2 \mathrm{~h}$ test for the different reinstatement conditions.

a

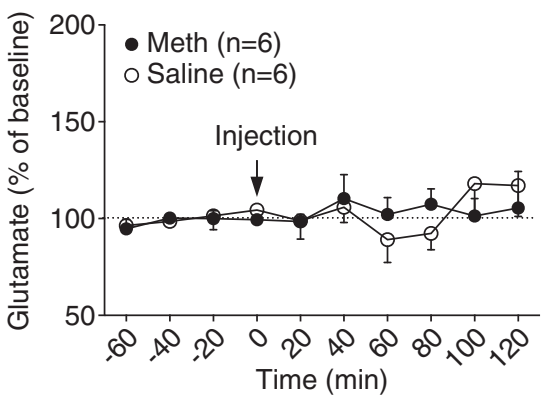

C

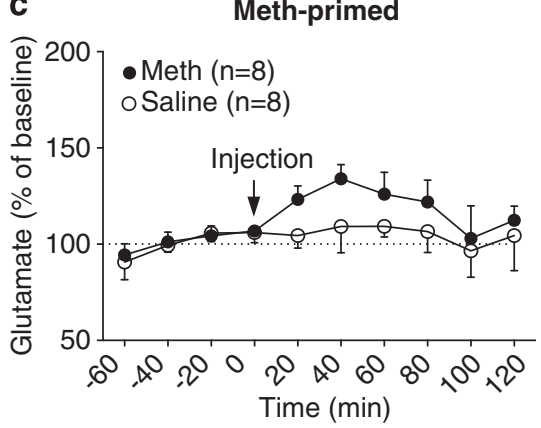

b

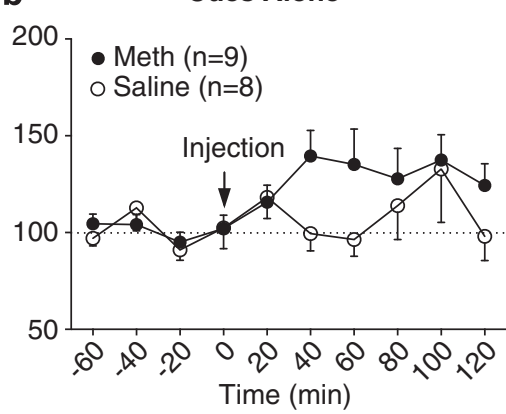

d

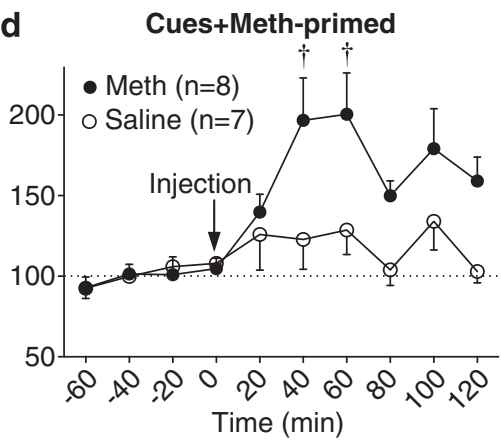

Figure 4 Extracellular GLU release in the dmPFC before and during reinstatement of meth seeking. Data are shown as \% of the baseline determined from the average of four baseline samples (time - 60 to 0) collected outside of the self-administration chamber. The arrow indicates either a saline or a meth prime $(1 \mathrm{mg} / \mathrm{kg})$ injection just before placement in the self-administration chamber. Stable baseline established before testing did not significantly change in either meth history or yoked-saline controls given a vehicle prime and no cues (a). There was a main effect of drug history in all three reinstatement conditions, as GLU efflux increased in the dmPFC of meth rats that experienced drug-associated cues and/or a meth prime (b-d). There was also a significant group $\times$ time interaction for cues + meth-primed reinstatement $(d)$, with significant differences found between meth and saline history rats at the 40 and 60 min time points $\left({ }^{\dagger} p<0.05\right)$.

seeking. Data are shown as the mean \pm SEM percentage of the baseline derived from the average of four baseline samples and were evaluated using a two-way RM ANOVA across time. No main effects of drug history, time, or the interaction were seen for the vehicle prime and no cues condition. For cue-induced reinstatement, there was a main effect of drug history $(\mathrm{F}(1,105)=5.91, p<0.05)$, but not for time, or for the interaction. For meth-primed reinstatement, there was also a main effect of drug history $(\mathrm{F}(1,140)=4.88$, $p<0.05$ ), but not a main effect of time or the interaction. Notably, for cues + meth-primed reinstatement, significant effects were found for drug history $(\mathrm{F}(1,130)=20.20$, $p<0.05)$, time $(\mathrm{F}(9,130)=6.23, p<0.05)$, and the interaction $(\mathrm{F}(9,130)=2.27, p<0.05)$. Rats with a meth history 

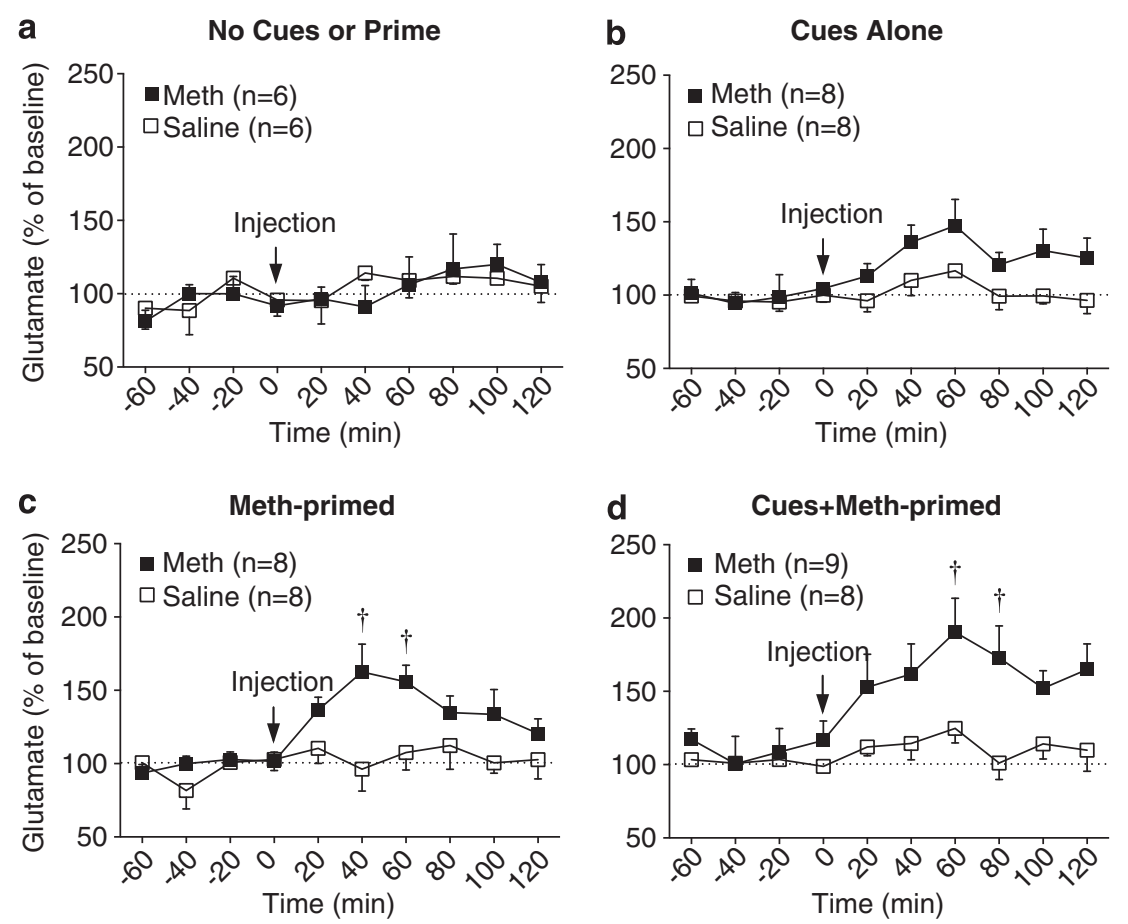

Figure 5 Extracellular GLU release in the NAc before and during reinstatement of meth seeking. Data are shown as \% of the baseline determined from the average of four baseline samples (time - 60 to 0) collected outside of the self-administration chamber. The arrow indicates either a saline or a meth prime $(\mathrm{I} \mathrm{mg} / \mathrm{kg}$ ) injection just before placement in the self-administration chamber. Stable baseline established before testing did not significantly change in either meth history or yoked-saline controls given a vehicle prime and no cues (a). There was a main effect of drug history in all three reinstatement conditions, as GLU efflux increased in the NAc of meth rats that experienced drug-associated cues and/or a meth prime (b-d). There were also significant group $\times$ time interactions for meth-primed (c) and cues + meth-primed (d) reinstatement, with significant differences found between meth and saline history rats at several time points $\left({ }^{\dagger} p<0.05\right)$.

showed robust GLU increases across the test, with pairwise comparisons showing significant differences from the yoked saline group at the 40 and 60 min time points $(p<0.05)$.

Figure 5 shows extracellular GLU in the NAc during (A) no cues or prime, (B) cue-induced, (C) meth-primed, or (D) cues + meth-primed reinstatement of meth seeking. No main effects of drug history or time, or a significant interaction were seen for the vehicle prime and no cues condition. For cue-induced reinstatement, there was a main effect of drug history $(\mathrm{F}(1,140)=14.40, p<0.05)$ and time $(\mathrm{F}(9,140)=3.00, p<0.05)$, but no significant interaction. However, for meth-primed reinstatement there was a main effect of drug history $(\mathrm{F}(1,140)=20.92, p<0.05)$ and time $(\mathrm{F}(9,140)=3.36, p<0.05)$, and the interaction was significant $(\mathrm{F}(9,140)=2.10, \quad p<0.05)$. Pairwise comparisons showed significant differences between meth and yokedsaline groups at the 40 and $60 \mathrm{~min}$ time points $(p<0.05)$. Finally, for cues + meth-primed reinstatement, significant effects were found for drug history $(\mathrm{F}(1,150)=29.29$, $p<0.05)$, time $(\mathrm{F}(9,150)=2.53, p<0.05)$, and the interaction $(\mathrm{F}(6,105)=2.32, \quad p<0.05)$. Pairwise comparisons showed significant differences between the treatment groups at the 60 and $80 \mathrm{~min}$ time points $(p<0.05)$.

\section{DA Efflux During Reinstatement}

DA levels were measured from the same sample collection vials as GLU. Figure 6 shows extracellular DA over time in the dmPFC during (A) no cues or prime, (B) cue-induced,
(C) meth-primed, or (D) cues + meth-primed reinstatement of meth seeking. No main effects of drug history, time, or the interaction were seen for the vehicle prime and no cues condition. For cue-induced reinstatement, there was no main effect of drug history, but there was a main effect of time $(\mathrm{F}(9,130)=2.30, p<0.05)$ and a significant interaction $(\mathrm{F}(9,130)=2.78, p<0.05)$. Pairwise comparisons showed a significant difference at the $40 \mathrm{~min}$ time point $(p<0.05)$. Notably, for meth-primed reinstatement, there were main effects of drug history $(\mathrm{F}(1,130)=9.31, p<0.05)$ and time $(\mathrm{F}(6,130)=2.00, p<0.05)$, and a significant interaction $(\mathrm{F}(6,130)=12.12, p<0.05)$. Pairwise comparisons between the meth self-administration and yoked-saline groups showed significant differences at the 60 and $80 \mathrm{~min}$ time points $(p<0.05)$. Finally, for cues + meth-primed reinstatement, there were significant main effects for drug history $(\mathrm{F}(1,140)=6.13, \quad p<0.05) \quad$ and time $(\mathrm{F}(9,140)=4.09$, $p<0.05)$, and a significant interaction $(\mathrm{F}(1,140)=2.03$, $p<0.05)$. Pairwise comparisons showed a significant difference at the $80 \mathrm{~min}$ time point $(p<0.05)$.

Figure 7 shows extracellular DA over time in the NAc during (A) no cues or prime, (B) cue-induced, (C) methprimed, or (D) cues + meth-primed reinstatement of meth seeking. No main effects of drug history, time, or the interaction were seen for the vehicle prime and no cues condition. For cue-induced reinstatement, there was a main effect of drug history $(\mathrm{F}(1,120)=12.12, p<0.05)$, but not for time or the interaction. Interestingly, for meth-primed reinstatement, there was a main effect of drug history 

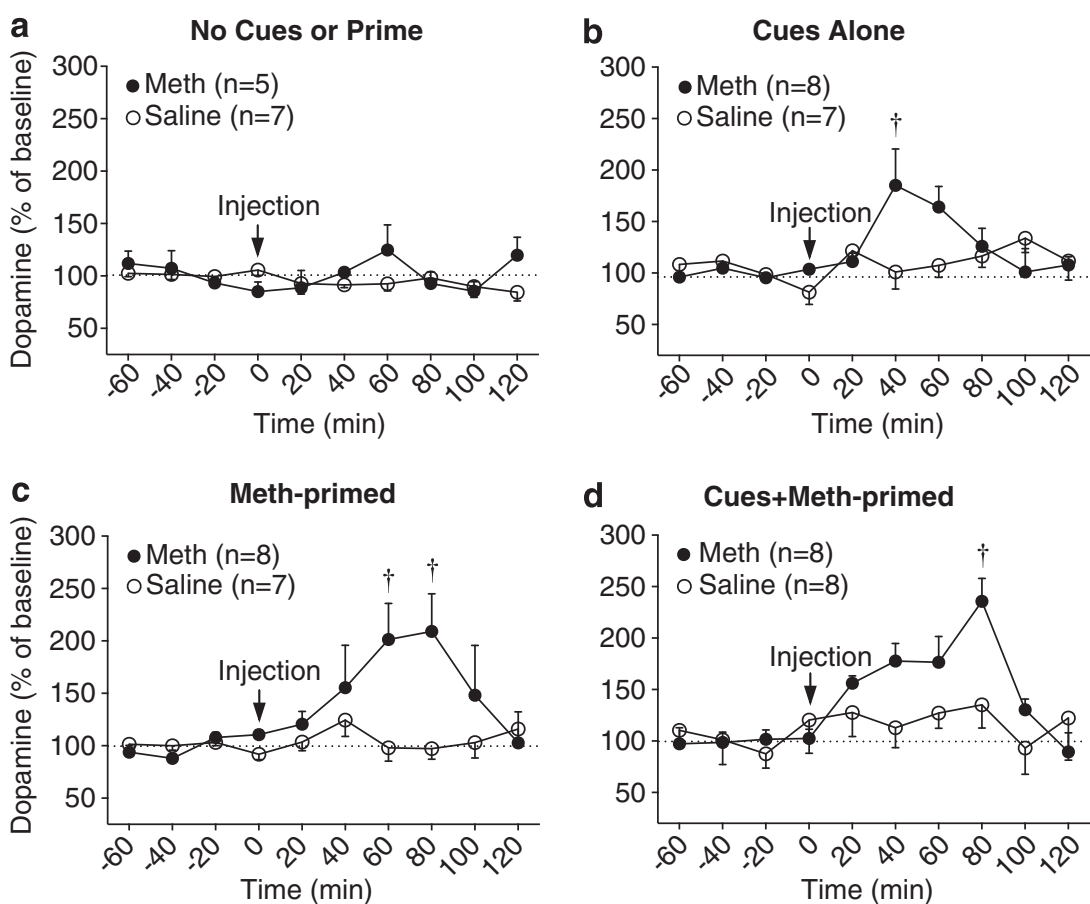

Figure 6 Extracellular DA release in the dmPFC before and during reinstatement of meth seeking. Data are shown as \% of the baseline determined from the average of four baseline samples (time -60 to 0) collected outside of the self-administration chamber. The arrow indicates either a saline or a meth prime $(\mathrm{I} \mathrm{mg} / \mathrm{kg})$ injection just before placement in the self-administration chamber. Stable baseline established before testing did not significantly change in either meth history or yoked-saline controls given a vehicle prime and no cues (a). There was a main effect of drug history and significant group $\times$ time interactions for all three reinstatement conditions $(b-d)$. Significant differences in DA efflux were seen between meth and saline history rats at several time points $(\uparrow p<0.05)$ under the different conditions of reinstatement.
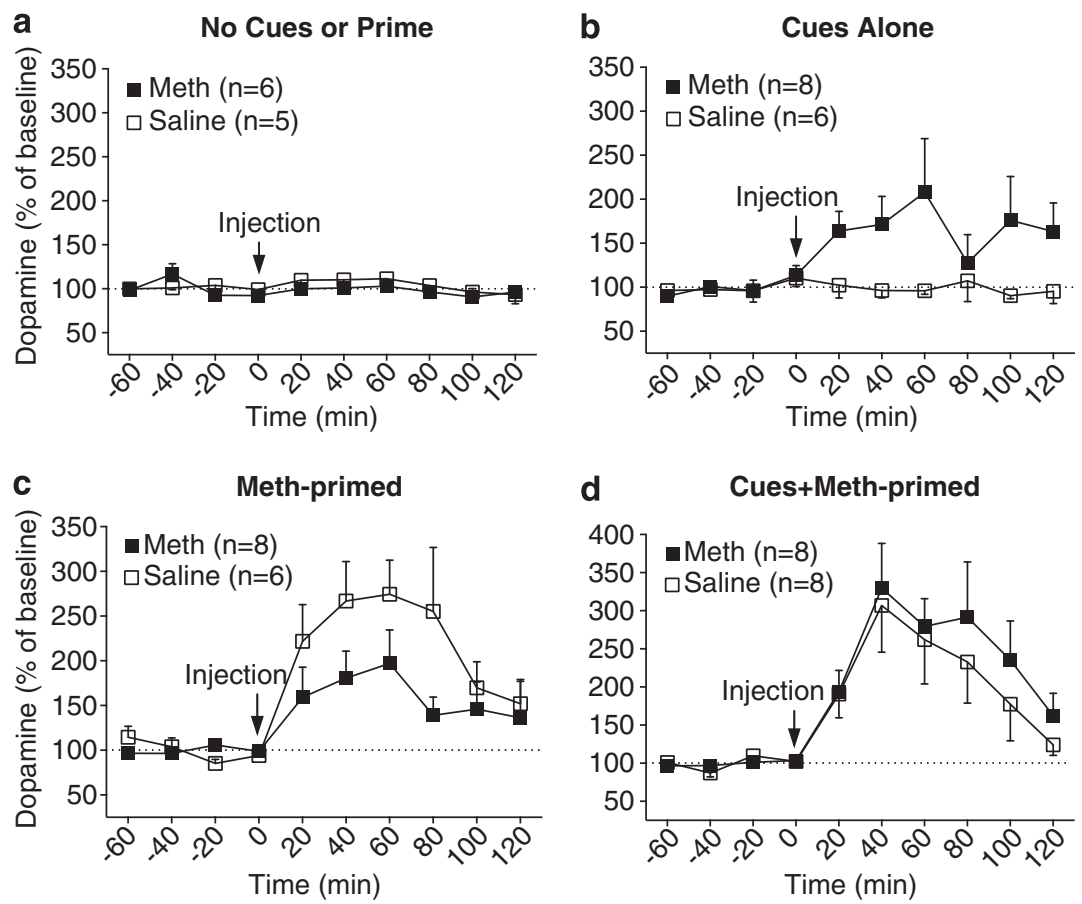

Figure 7 Extracellular DA release in the NAc before and during reinstatement of meth seeking. Data are shown as \% of the baseline determined from the average of four baseline samples (time - 60 to 0) collected outside of the self-administration chamber. The arrow indicates either a saline or a meth prime $(\mathrm{l} \mathrm{mg} / \mathrm{kg})$ injection just before placement in the self-administration chamber. Stable baseline established before testing did not significantly change in either meth history or yoked-saline controls given a vehicle prime and no cues (a). There were main effects of drug history for cue-induced reinstatement (b), drug history and time for meth-primed reinstatement (c), and time for cues + meth-primed reinstatement (d). 
$(\mathrm{F}(1,120)=8.18, p<0.05)$ and for time $(\mathrm{F}(9,120)=6.67$, $p<0.05)$, with a trend for increased DA efflux to be lower in rats with a meth history. However, the interaction was not significant. Finally, for cues + meth-primed reinstatement, a robust increase in DA release occurred in both groups, with a significant main effect for time $(\mathrm{F}(9,140)=9.89, p<0.05)$, but not for drug history or the interaction.

\section{DISCUSSION}

Previous studies using self-administration/reinstatement models have focused primarily on drug-induced changes in GLU and DA regulation within the NAc. Here, we demonstrated that a history of meth self-administration reduced basal GLU levels not only in the NAc core but also in its key projection source, the dmPFC. In addition, reinstatement of meth seeking triggered by meth-associated cues in combination with a meth prime increased extracellular GLU in both the dmPFC and NAc only in rats with meth self-administration experience. Notably, increased extracellular GLU during reinstatement paralleled the active lever pressing, particularly in the cues + meth-primed reinstatement condition. Our results also show that DA efflux in the dmPFC, in contrast to the NAc, robustly increased under all reinstatement conditions only in rats who had previously self-administered meth. These data thus reveal that meth-induced GLU dysregulation clearly involves changes in GLU efflux within the cortical efferent projection region of the corticostriatal pathway and that hyper activation of both GLU and DA efflux in the dmPFC occurs during reinstatement of meth seeking.

\section{Reduced Basal GLU after Meth Self-Administration}

GLU transmission in the corticostriatal pathway has been identified as critical in the expression of drug seeking at the time of reinstatement (McFarland et al, 2003; Rocha and Kalivas, 2010). Furthermore, the relationship between extrasynaptic and synaptic basal GLU in the NAc has been explored for its role in mediating drug seeking (Baker et al, 2003; Cornish and Kalivas, 2000; Kalivas, 2009). However, the present data also show concurrent reductions in basal GLU in both the dmPFC and the NAc in subjects with a history of meth self-administration. Human neuroimaging studies of meth addicts have reported the loss of both white and gray matter as well as decreased metabolism (Berman et al, 2008; Kim et al, 2005) in prefrontal areas that are analogous to the dmPFC in the rat (Ongür and Price, 2000; Uylings et al, 2003). These meth-induced cortical disruptions may reduce the ability to regulate subcortical drives in limbic structures, including the NAc (Goldstein et al, 2009). Consistent with this hypothesis, human meth addicts often exhibit enduring PFC-dependent cognitive deficits in tasks requiring attention, working memory, and suppression of irrelevant information (Baicy and London, 2007; Salo et al, 2009), as well as enduring downregulation of DA transporter (DAT) function in the frontal cortex, NAc, and caudate/putamen (Chang et al, 2007; McCann et al, 2008).

Reductions in basal NAc GLU following cocaine selfadministration have been attributed to decreased affinity of the cystine-GLU exchanger (Baker et al, 2003; McFarland et al, 2003). Restoration with the GLU prodrug, N-acetylcysteine (either systemic or intra NAc), can prevent reinstatement following a cocaine priming injection (Baker et al, 2003). The current findings not only show a similar meth-induced reduction in basal NAc GLU but also indicate that reduced basal cortical GLU levels likely contribute to the maladaptive balance in synaptic and extrasynaptic extracellular GLU activity in the corticostriatal pathway. Interestingly, a recent finding by Lominac et al (2012) reported that rats with a similar $2 \mathrm{~h}$ /day meth self-administration history followed by withdrawal showed an increase in basal NAc GLU levels. Notably, they measured GLU efflux following simple withdrawal at 1 day and 3 weeks, not after daily extinction sessions. Drug-induced neuroadaptations after extinction $v s$ forced abstinence (withdrawal) have been shown to differ in many respects, including the specific neural regions involved (Fuchs et al, 2006) and changes in GLU receptor subtype levels (Schwendt et al, 2012). Thus, the nature of the withdrawal experience may help explain different findings for NAc GLU efflux.

\section{Increased GLU Efflux during Meth Seeking}

In contrast to decreased basal metabolic function, neuroimaging studies in psychostimulant dependent subjects have consistently reported that upon exposure to drug-related cues or low doses of psychostimulants, metabolic activity increases in the anterior cingulate, dorsolateral prefrontal cortex (PFC), and NAc (Bonson et al, 2002; Volkow et al, 2005). Our results showed that GLU efflux increased in both the dmPFC and NAc during cue-induced and meth-primed reinstatement. The increase in NAc GLU efflux seen during meth-primed reinstatement is consistent with previous findings with cocaine (McFarland et al, 2003). Notably, the most pronounced GLU efflux in both the dmPFC and NAc occurred during the cues + meth-primed reinstatement, suggesting that the two modalities of cues and a meth prime combination are additive as reflected by increased meth seeking. Although we do not currently have direct evidence, it is possible that the enhanced GLU release under combined cues + meth-primed reinstatement results from multiple sources of neuronal GLU. The dmPFC projection to the NAc is regulated by a number of projections, including the mesocorticolimbic DA projection from the VTA, and GLU inputs from the hippocampus, bed nucleus of the stria terminalis, and the amygdala. The GLU projections are especially relevant as they contribute information about the organism's internal state and ongoing information processing (Kelly and Strick, 2004; Reynolds and Zahm, 2005) and may be more critical for cue-induced, relative to drug-primed reinstatement of drug seeking (See, 2005).

\section{Increased DA Efflux during Meth Seeking}

In the dmPFC, DA efflux consistently increased across all reinstatement conditions, but only in meth self-administering rats. Pharmacological inactivation of the dmPFC blocks reinstatement of both cocaine (McFarland et al, 2003) and meth (Rocha and Kalivas, 2010) seeking, but does not block DA release in the NAc (McFarland et al, 2003). Notably, 
direct infusion of DA or cocaine into the $\mathrm{dmPFC}$ reinstates cocaine seeking (McFarland and Kalivas, 2001; Park et al, 2002), whereas dmPFC DA receptor blockade blocks reinstatement (Sun and Rebec, 2005). In contrast, DA receptor blockade in the NAc core does not prevent reinstatement of cocaine seeking (McFarland and Kalivas, 2001). Taken together with the current data showing increased DA efflux in the dmPFC during reinstatement of meth seeking, dmPFC DA clearly has a key role in driving reinstatement of drug seeking, perhaps by directly activating DA receptors located on the cortical GLU projection neurons to the NAc.

The lack of effect of acute meth on dmPFC DA release in yoked-saline subjects was not due to the meth dose, as these subjects showed a robust increase in NAc DA over time. In fact, when meth was administered in the absence of cues, yoked subjects showed evidence of a greater NAc DA response than rats with a meth self-administration history. Interestingly, a few of the yoked-control subjects that modestly responded on both levers during reinstatement showed no evidence of increased dmPFC DA (data not shown). This lack of change in DA efflux suggests that increased dmPFC DA during reinstatement may be particularly related to meth-paired associations and not simply pharmacologically driven DA release.

Increased DA signaling is known to be important for encoding the motivational value of reward-predicting cues. One view is that phasic DA signal provides a 'prediction error,' being initially activated by an unconditioned reward itself (eg, meth) and through repeated experience eventually transitions to signal the presence of the reward-predicting stimuli (Schultz, 2007). Moreover, phasic increases in DA neuron firing have been shown to reflect how predictive the learned associations match the expected reward to the reward outcome (Waelti et al, 2001). Thus, as seen in the current study, a larger than expected reward (meth prime) or the unexpected CS presentation during reinstatement may produce greater VTA activation and subsequent cortical DA release. Interestingly, the combined cues + meth-primed condition did not elicit a further increase of dmPFC DA, suggesting that these two modalities are not additive in regards to DA efflux. This may be due to either feedback GLU projections from the PFC onto VTA interneurons, which can attenuate subsequent cortical DA release (Carr and Sesack, 2000) and/or the far fewer DA projections from the VTA to the $\mathrm{dmPFC}$, relative to the NAc.

DA signaling has alternatively been proposed to confer learned reward cues with incentive salience (Berridge and Robinson, 1998). In this view, increased DA is not necessary for all forms of learning but is critical for imbuing rewardrelated cues with motivational significance (Flagel et al, 2011). As such, reward-associated stimuli that elicit phasic DA release also initiate reward seeking or invigorate ongoing behavior (eg, lever pressing), even in the absence of the reward itself (Berridge and Robinson, 1998). Psychostimulant-induced increases in DA facilitate conditioned reinforcing effects of both food and drug-associated cues to elicit these behaviors (Kelley and Delfs, 1991; Taylor and Robbins, 1984), thus reinforcing drug seeking and the transition from abuse to addiction (Robinson and Berridge,
2003; Wise, 2004). Consistently, our data showed increased DA efflux during exposure to the reward-predicting stimuli (eg, drug prime or drug-associated cues), even in the absence of the drug itself (eg, cues only condition), which predictably corresponded with meth seeking. As DA efflux increased in the dmPFC, but not in the NAc under each reinstatement condition, DA release specifically in the dmPFC may constitute a common mechanism for meditating reinstatement of meth seeking.

\section{Potential Mechanisms in PFC-NAc Regulation of Meth Seeking}

The current data indicate that neurotransmitter release in the PFC may exhibit unique patterns under varied conditions of cue-induced and meth-primed reinstatement. Specifically, we observed that DA and GLU release differed somewhat in the PFC under each modality of reinstatement, even though drug seeking was similar. This may reflect the initiation of drug seeking through distinct neurochemical activation within the PFC that subsequently influences events downstream in the NAc. One possibility is that variations in the temporal nature of PFC DA release during cue presentations $v$ s meth prime alone differentially activate PFC DA receptors, resulting in unique efflux patterns under each reinstatement condition. Cues likely elicit more acute PFC DA efflux, whereas a meth prime maintains a longer cumulative increase in PFC DA signaling. This differential pattern is consistent with the results of GLU efflux measured in the NAc during the two forms of reinstatement. Cue presentations alone more rapidly increased PFC DA, but only moderately increased NAc GLU release. Conversely, the meth prime produced slower increases in PFC DA, but resulted in greater NAc GLU release. Notably, PFC GLU, but not DA, increased more when meth-associated cues and meth prime conditions were combined, as compared with either modality alone. It is possible that each modality contributes GLU input to the PFC from independent sources, thereby enhancing drug seeking in an additive manner. For example, cue-induced reinstatement may rely to a greater extent on amygdalar glutamatergic inputs to both the PFC and NAc (Chefer et al, 2011; Stuber et al, 2011).

As in the PFC, the temporal nature of the drug-cue presentations, as compared with meth prime, may be important for the pattern of corticoaccumbens GLU release. It has been shown that learned drug-associated (but not sucrose-associated) cues can induce rapid, transient PFCactivity-dependent changes in spine morphology in the NAC of rats with a history of cocaine self-administration. Furthermore, greater PFC-dependent transient plasticity in the NAc corresponded with a higher intensity of drug seeking (Gipson et al, 2013). Taken with the current findings, this suggests that cues may promote drug seeking through more distinct quantitative neurochemical accumulation, whereas a drug prime more broadly increases overall neurochemical tone. Under this scenario, combining the two modalities leads to additive increases in efflux and corresponding increases in drug seeking.

The PFC possesses more excitatory D1 (Gs-coupled) mRNA and D1 receptor-binding sites than any other DA receptor subtypes (Gaspar et al, 1995; Vincent et al, 1993). 
Most of these receptors are located on pyramidal neurons that project to other regions, including the NAc (Santana et al, 2009; Vincent et al, 1993). Importantly, DA D1 receptor activation in the dmPFC has been identified as necessary for drug seeking (Capriles et al, 2003; Sun and Rebec, 2005), and direct infusion of DA, cocaine, amphetamine, or selective DA agonists into the dmPFC reinstates drug seeking (McFarland and Kalivas, 2001) and increases GLU in the NAc (Park et al, 2002). An additional mechanism may occur after chronic elevation of dmPFC DA by meth, whereby peri/extrasynaptic inhibitory D2-like (Gi-coupled) autoreceptors compensate for the excessive DA signaling. As a consequence, D2 receptors could become downregulated, perhaps through increased expression of G-protein signaling 3 (Bowers et al, 2004), eventually favoring D1 receptor activation. During reinstatement, increased dmPFC DA efflux would primarily activate the excitatory D1 receptors, including those on pyramidal neurons projecting to the NAc, with a subsequent increase in GLU release. Consistent with this idea, neuroimaging data in human addicts have shown decreased Gi $\alpha$ coupled receptors (such as D2) in the PFC regions, including the anterior cingulate (Goldstein et al, 2009).

Finally, it should be emphasized that a balance of extrasynaptic and synaptic GLU function in the NAc mediates cocaine seeking (Baker et al, 2003; Kalivas, 2009). Although similar mechanisms that regulate extracellular GLU exist in both the PFC and NAc (including the cystine-GLU exchanger, excitatory amino-acid transporters, and several GLU receptor subtypes), these proteins may regulate extracellular GLU in ways that differ from striatal regions (Melendez et al, 2005). Further, the dmPFC receives its major GLU inputs from disparate cortical and subcortical sources, thus making precise estimate of extrasynaptic regulation more difficult to assess. Notably, a recent report showed that systemic treatment with the GLU prodrug, ceftriaxone, effectively blocked meth conditioned place preference and upregulated the GLU transporter in the PFC, but not the NAc (Abulseoud et al, 2012). In addition, a selective decrease in mGluR $2 / 3$ receptors was found in the PFC, but not in the NAc or caudate/putamen, following chronic meth self-administration and extinction (Schwendt et al, 2012). Thus, it will be important to further determine the unique regional changes in the various mechanisms of GLU function that result from repeated meth self-administration.

\section{CONCLUSIONS}

In summary, the current findings demonstrate that a history of meth self-administration leads not only to decreased basal GLU in the NAc but also to decreased basal GLU in the primary neuronal source of striatal GLU, namely the dmPFC. Furthermore, chronic meth intake leads to enhancement of both cortical GLU and DA release during exposure to drug-associated cues and a meth priming injection in a manner not seen in drug naïve subjects. On the basis of the critical role of the corticoaccumbens pathway in drug addiction, it will be important to further explore how these alterations in extracellular GLU and DA release within the dmPFC relate to intracortical protein changes induced by chronic meth self-administration. In addition, given the critical role of stress factors in drug addiction, the role of PFC GLU and DA in stress-induced reinstatement of meth seeking deserves future exploration. Identifying appropriate neuronal targets in these models will be an important step in understanding the maladaptive changes underlying meth addiction and directing potential future treatments.

\section{FUNDING AND DISCLOSURE}

The authors declare no conflict of interest.

\section{ACKNOWLEDGEMENTS}

We thank Brittney Cox, Andrew Novak, Shannon Ghee, and Clifford Chan for their technical assistance. This research was supported by National Institute on Drug Abuse (NIDA) grants DA016511, DA033049, and DA029431, and NIH grant C06 RR015455.

\section{Author Contributions}

AP designed and performed the experiments and data analysis, and wrote the manuscript; RES designed, supervised, supported the project, and wrote the manuscript.

\section{REFERENCES}

Abulseoud OA, Miller JD, Wu J, Choi D-S, Holschneider DP (2012). Ceftriaxone upregulates the glutamate transporter in medial prefrontal cortex and blocks reinstatement of methamphetamine seeking in a condition place preference paradigm. Brain Res 1456: 14-21.

Baicy K, London ED (2007). Corticolimbic dysregulation and chronic methamphetamine abuse. Addiction 102(Suppl 1): 5-15.

Baker D, McFarland K, Lake R, Shen H (2003). Neuroadaptations in cystine-glutamate exchange underlie cocaine relapse. Nature 6: 743-749.

Berman SM, Voytek B, Mandelkern MA, Hassid BD, Isaacson A, Monterosso J et al (2008). Changes in cerebral glucose metabolism during early abstinence from chronic methamphetamine abuse. Mol Psychiatry 13: 897-908.

Berridge KC, Robinson TE (1998). What is the role of dopamine in reward: hedonic impact, reward learning, or incentive salience? Brain Res Brain Res Rev 28: 309-369.

Bonson KR, Grant SJ, Contoreggi CS, Links JM, Metcalfe J, Weyl HL et al (2002). Neural systems and cue-induced cocaine craving. Neuropsychopharmacology 26: 376-386.

Bowers MS, McFarland K, Lake R, Peterson Y (2004). Activator of $G$ protein signaling 3: a gatekeeper of cocaine sensitization and drug seeking. Neuron 42: 269-281.

Bowyer JF, Robinson B, Ali S, Schmued LC (2008). Neurotoxicrelated changes in tyrosine hydroxylase, microglia, myelin, and the blood-brain barrier in the caudate-putamen from acute methamphetamine exposure. Synapse 62: 193-204.

Capriles N, Rodaros D, Sorge RE (2003). A role for the prefrontal cortex in stress-and cocaine-induced reinstatement of cocaine seeking in rats. Psychopharmacology 168: 66-74.

Carr DB, Sesack SR (2000). Projections from the rat prefrontal cortex to the ventral tegmental area: target specificity in the synaptic associations with mesoaccumbens and mesocortical neurons. J Neurosci 20: 3864-3873. 
Chang L, Alicata D, Ernst T, Volkow N (2007). Structural and metabolic brain changes in the striatum associated with methamphetamine abuse. Addiction 102(Suppl 1): 16-32.

Chefer VI, Wang R, Shippenberg TS (2011). Basolateral amygdaladriven augmentation of medial prefrontal cortex GABAergic neurotransmission in response to environmental stimuli associated with cocaine administration. Neuropsychopharmacology 36: 2018-2029.

Cornish JL, Kalivas PW (2000). Glutamate transmission in the nucleus accumbens mediates relapse in cocaine addiction. J Neurosci 20: RC89.

Di Chiara G (1999). Drug addiction as dopamine-dependent associative learning disorder. Eur J Pharmacol 375: 13-30.

Flagel SB, Clark JJ, Robinson TE, Mayo L, Czuj A, Willuhn I et al (2011). A selective role for dopamine in stimulus-reward learning. Nature 469: 53-57.

Fowler J, Volkow N, Logan J, Alexoff D (2008). Fast uptake and long-lasting binding of methamphetamine in the human brain: comparison with cocaine. NeuroImage 43: 756-763.

Fuchs RA, Evans AK, Parker MP, See RE (2004). Differential involvement of orbitofrontal cortex subregions in conditioned cue-induced and cocaine-primed reinstatement of cocaine seeking in rats. J Neurosci 24: 6600-6610.

Fuchs RA, Branham RK, See RE (2006). Different neural substrates mediate cocaine seeking after abstinence versus extinction training: a critical role for the dorsolateral caudate-putamen. J Neurosci 26: 3584-3588.

Gaspar P, Bloch B, Le Moine C (1995). D1 and D2 receptor gene expression in the rat frontal cortex: cellular localization in different classes of efferent neurons. Eur J Neurosci 7: 1050-1063.

George O, Koob GF (2010). Individual differences in prefrontal cortex function and the transition from drug use to drug dependence. Neurosci Biobehav Rev 35: 232-247.

Gipson CD, Kupchik YM, Shen H, Reissner KJ, Thomas CA, Kalivas PW (2013). Relapse induced by cues predicting cocaine depends on rapid, transient synaptic potentiation. Neuron 77: 867-872.

Goldstein RZ, Craig ADB, Bechara A, Garavan H, Childress AR, Paulus MP et al (2009). The neurocircuitry of impaired insight in drug addiction. Trends Cogn Sci 13: 372-380.

Haughey HM, Brown JM, Wilkins DG, Hanson GR, Fleckenstein AE (2000). Differential effects of methamphetamine on $\mathrm{Na}(+) / \mathrm{Cl}(-)-$ dependent transporters. Brain Res 863: 59-65.

Kalivas PW (2009). The glutamate homeostasis hypothesis of addiction. Nat Rev Neurosci 10: 561-572.

Kalivas PW, Volkow ND, Seamans J (2005). Unmanageable motivation in addiction: a pathology in prefrontal-accumbens glutamate transmission. Neuron 45: 647-650.

Kelley AE, Delfs JM (1991). Dopamine and conditioned reinforcement. I. Differential effects of amphetamine microinjections into striatal subregions. Psychopharmacology 103: 187-196.

Kelly RM, Strick PL (2004). Macro-architecture of basal ganglia loops with the cerebral cortex: use of rabies virus to reveal multisynaptic circuits. Prog Brain Res 143: 449-459.

Kim SJ, Lyoo IK, Hwang J, Sung YH, Lee HY, Lee DS et al (2005). Frontal glucose hypometabolism in abstinent methamphetamine users. Neuropsychopharmacology 30: 1383-1391.

Lominac KD, Sacramento AD, Szumlinski KK, Kippin TE (2012). Distinct neurochemical adaptations within the nucleus accumbens produced by a history of self-administered vs non-contingently administered intravenous methamphetamine. Neuropsychopharmacology 37: 707-722.

Lonnroth P, Jansson PA, Smith U (1987). A microdialysis method allowing characterization of intercellular water space in humans. Am J Physiol 253: E228-E231.

McCann UD, Kuwabara H, Kumar A, Palermo M, Abbey R, Brasic J et al (2008). Persistent cognitive and dopamine transporter deficits in abstinent methamphetamine users. Synapse 62: 91-100.
McFarland K, Kalivas PW (2001). The circuitry mediating cocaineinduced reinstatement of drug-seeking behavior. J Neurosci 21: $8655-8663$

McFarland K, Lapish CC, Kalivas PW (2003). Prefrontal glutamate release into the core of the nucleus accumbens mediates cocaineinduced reinstatement of drug-seeking behavior. J Neurosci 23: 3531-3537.

McLaughlin J, See RE (2003). Selective inactivation of the dorsomedial prefrontal cortex and the basolateral amygdala attenuates conditioned-cued reinstatement of extinguished cocaine-seeking behavior in rats. Psychopharmacology 168: 57-65.

Melendez R, Vuthiganon J, Kalivas P (2005). Regulation of extracellular glutamate in the prefrontal cortex: focus on the cystine glutamate exchanger and group I metabotropic glutamate receptors. J Pharmacol Exp Ther 314: 139-147.

Ongür D, Price JL (2000). The organization of networks within the orbital and medial prefrontal cortex of rats, monkeys and humans. Cereb Cortex 10: 206-219.

Park W-K, Bari AA, Jey AR, Anderson SM, Spealman RD, Rowlett JK et al (2002). Cocaine administered into the medial prefrontal cortex reinstates cocaine-seeking behavior by increasing AMPA receptor-mediated glutamate transmission in the nucleus accumbens. J Neurosci 22: 2916-2925.

Paxinos G, Watson C (1997). The Rat Brain in Stereotaxic Coordinates. 3rd edn. Academic Press: New York, NY, USA.

Reichel CM, Moussawi K, Do PH, Kalivas PW, See RE (2011). Chronic N-acetylcysteine during abstinence or extinction after cocaine self-administration produces enduring reductions in drug seeking. J Pharmacol Exp Ther 337: 487-493.

Reynolds SM, Zahm DS (2005). Specificity in the projections of prefrontal and insular cortex to ventral striatopallidum and the extended amygdala. J Neurosci 25: 11757-11767.

Robinson TE, Berridge KC (2003). Addiction. Ann Rev Psychol 54: 25-53.

Rocha A, Kalivas PW (2010). Role of the prefrontal cortex and nucleus accumbens in reinstating methamphetamine seeking. Eur J Neurosci 31: 903-909.

Salo R, Ursu S, Buonocore M, Leamon M (2009). Impaired prefrontal cortical function and disrupted adaptive cognitive control in methamphetamine abusers: a functional magnetic resonance imaging study. Biol Psychiatry 65: 706-709.

Santana N, Mengod G, Artigas F (2009). Quantitative analysis of the expression of dopamine D1 and D2 receptors in pyramidal and GABAergic neurons of the rat prefrontal cortex. Cereb Cortex 19: 849-860.

Schultz W (2007). Behavioral dopamine signals. Trends Neurosci 30: $203-210$

Schwendt M, Reichel CM, See RE (2012). Extinction-dependent alterations in corticostriatal mGluR2/3 and mGluR7 receptors following chronic methamphetamine self-administration in rats. PLoS One 7: e34299.

See R (2005). Neural substrates of cocaine-cue associations that trigger relapse. Eur J Pharmacol 526: 140-146.

Shoblock JR, Sullivan EB, Maisonneuve IM, Glick SD (2003). Neurochemical and behavioral differences between d-methamphetamine and d-amphetamine in rats. Psychopharmacology 165 : 359-369.

Stephans SE, Yamamoto BK (1994). Methamphetamine-induced neurotoxicity: roles for glutamate and dopamine efflux. Synapse 17: 203-209.

Stuber GD, Sparta DR, Stamatakis AM, van Leeuwen WA, Hardjoprajitno JE, Cho S et al (2011). Excitatory transmission from the amygdala to nucleus accumbens facilitates reward seeking. Nature 475: 377-380.

Sun WL, Rebec GV (2005). The role of prefrontal cortex D1-like and D2-like receptors in cocaine-seeking behavior in rats. Psychopharmacology 177: 315-323. 
Dopamine and glutamate after methamphetamine in rats A Parsegian and RE See

Taylor JR, Robbins TW (1984). Enhanced behavioural control by conditioned reinforcers following microinjections of d-amphetamine into the nucleus accumbens. Psychopharmacology 84: 405-412.

Uylings HB, Groenewegen HJ, Kolb B (2003). Do rats have a prefrontal cortex? Behav Brain Res 146: 3-17.

Vincent SL, Khan Y, Benes FM (1993). Cellular distribution of dopamine D1 and D2 receptors in rat medial prefrontal cortex. J Neurosci 13: 2551-2564.
Volkow N, Wang G, Ma Y, Fowler J, Wong C, Ding YS et al (2005). Activation of orbital and medial prefrontal cortex by methylphenidate in cocaine-addicted subjects but not in controls: relevance to addiction. J Neurosci 25: 3932-3939.

Waelti P, Dickinson A, Schultz W (2001). Dopamine responses comply with basic assumptions of formal learning theory. Nature 412: 43-48.

Wise R (2004). Dopamine, learning and motivation. Nat Rev Neurosci 5: 483-494. 